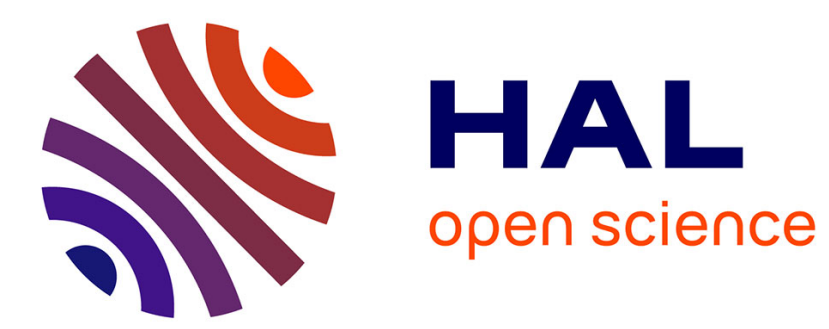

\title{
La révision de vie
}

Anthony Favier

\section{To cite this version:}

Anthony Favier. La révision de vie. Archives de Sciences Sociales des Religions, 2019, Conversions à l'islam, culture et religion. Tensions et articulations., 186, pp.141-162. 10.4000/assr.45726 . halshs02129615

\section{HAL Id: halshs-02129615 https://shs.hal.science/halshs-02129615}

Submitted on 15 May 2019

HAL is a multi-disciplinary open access archive for the deposit and dissemination of scientific research documents, whether they are published or not. The documents may come from teaching and research institutions in France or abroad, or from public or private research centers.
L'archive ouverte pluridisciplinaire HAL, est destinée au dépôt et à la diffusion de documents scientifiques de niveau recherche, publiés ou non, émanant des établissements d'enseignement et de recherche français ou étrangers, des laboratoires publics ou privés. 


\section{Anthony Favier}

\section{La révision de vie}

\section{Une pratique religieuse méconnue au cœur du catholicisme français}

Si les pratiques religieuses peuvent susciter l'intérêt des historiens médiévistes et modernistes, l'histoire religieuse contemporaine, contrairement à la sociologie ${ }^{1}$ ou à l'anthropologie (Piette, 1999: 271), s'est peu penchée sur la question. En France, la focalisation sur la sécularisation, notamment à travers l'étude minutieuse de l'érosion démographique dans l'assistance aux sacrements ${ }^{2}$, s'est effectuée au détriment des autres pratiques développées par ceux qui restent croyants. Cela est particulièrement vrai pour la révision de vie en usage dans les mouvements de laïcs français à partir des années 1930.

Ce relatif silence de l'historiographie est d'autant plus surprenant que de nombreuses études renouvellent aujourd'hui l'intérêt pour les formes religieuses qui ne se réduisent pas à l'assistance au culte dominical. En témoignent de récents travaux sur le sacrement de la pénitence (Cuchet, 2015) ou la direction de conscience (Muller, 2019). D’un point de vue plus large, la pensée de Michel Foucault, qui occupe une place essentielle dans les sciences humaines et sociales contemporaines, aurait également pu aiguiller l'attention des chercheurs. En effet, elle met en lumière l'importance des exercices que le sujet réalise pour parvenir à la vérité sur soi. Dans son travail, le philosophe a rapidement pointé du doigt le rôle des chrétiens, avant même la période moderne, dans ce processus (Foucault, 2018). Or, la révision de vie n'a pas retenu l'attention des chercheurs travaillant sur l'époque contemporaine.

La pratique dont nous allons traiter ici appartient, en réalité, à un éventail de dispositifs de relecture, par un croyant, d'événements biographiques à la

\footnotetext{
1. Par exemple, pour Danièle Hervieu-Léger, dans l'étude du religieux en contexte contemporain, «il faut pousser la démarche jusqu'à l'analyse des structures et de la dynamique du croire religieux moderne, en y incluant [...] non seulement les “croyances", qui sont les objets idéels des convictions individuelles et collectives, mais l'ensemble des pratiques [...] dans lesquelles ces croyances prennent corps» (1993: 9).

2. En 2011 s'achevait la publication du dernier volume des Matériaux pour l'histoire religieuse du peuple français, plus connu sous le nom de Matériaux Boulard. Sur cette approche scientifique déterminante des sciences religieuses du catholicisme français, voir Sorrel, 2013: 193.
} 
lumière des vérités religieuses. En un sens, la révision de vie se rapproche de la retraite, de la récollection ou de l'examen de conscience sans toutefois s'y réduire. La pratique a eu un certain succès au sein du catholicisme français et occidental au xx $x^{\mathrm{e}}$ siècle. Elle naît dans l'entre-deux-guerres, au sein de la Jeunesse ouvrière chrétienne (JOC), et se répand en France grâce à l'action des aumôniers, dans les années 1950-1960, avec les mouvements d'Action catholique $^{3}$. La révision de vie a nourri une vision du monde selon laquelle le quotidien, puis, progressivement, les événements contemporains pouvaient être interprétés comme le témoignage de l'accomplissement des promesses évangéliques. Instrument ou symptôme d'une politisation de certains courants du catholicisme français dans les années 1970, la révision de vie n'a pas disparu depuis, même si elle a perdu en centralité dans les discours des acteurs pastoraux ou des mouvements de laïcs.

Cet article envisage la révision de vie comme une pratique sociale à la croisée d'enjeux laïques et religieux multiples. À travers son étude, on peut réfléchir sur la façon dont naissent, se développent et disparaissent des pratiques sociales et religieuses dans un contexte particulier. Pourquoi la révision de vie a-t-elle été au cœur du catholicisme des années 1940-1970 avant de connaître un relatif déclin? A-t-elle été un lieu d'amenuisement, de déchargement ou de recomposition du religieux?

\section{Du “cercle d'étude " à la "révision de vie " (des origines de la JOC-F aux années 1940)}

La Jeunesse ouvrière chrétienne 4 est créée en Belgique par le prêtre Joseph Cardijn en 1925. Elle est importée en France, avec quelques adaptations, grâce aux jésuites engagés dans le champ du catholicisme social, par un vicaire d'une paroisse ouvrière du diocèse de Paris, Georges Guérin, entre 1926 et 1928 (Cholvy, 2000). Les premiers manuels à destination des aumôniers et des cadres ne mentionnent pas la révision de vie. L'apport décisif de la JOC vient alors plutôt de sa méthode réflexive d'action. Lexicalisé sous le slogan «voir, juger, agir », le principe a été théorisé par Joseph Cardijn lui-même, à la fin des années 1920, sous une forme originellement passive: «la méthode jociste [...] se résume en trois choses élémentaires: faire voir, faire juger, faire agir » ${ }^{5}$.

3. L'Action catholique désigne une famille particulière de mouvements de laïcs catholiques qui s'organisent au début du $\mathrm{XX}^{\mathrm{e}}$ siècle sur les indications du pape Pie X et se spécialisent par «milieux» dans l'entre-deux-guerres avec Pie XI. On y valorise l'autonomie des militants, qui doivent devenir les relais des clercs dans la société, ainsi que la recherche de réponses concrètes aux problèmes sociaux contemporains dans le sens de la doctrine catholique.

4. Dans ce travail, seront abrégées sous l'acronyme JOC la Jeunesse ouvrière chrétienne (mouvement masculin) et sous la forme JOCF la Jeunesse ouvrière chrétienne féminine. Lorsque je désignerai les deux, c'est-à-dire JOCF et JOC, j'utiliserai le sigle JOC-F.

5. «Conférence de Monsieur l'abbé Cardijgn [sic] fondateur de la Jeunesse ouvrière chrétienne belge JOCB donnée au séminaire d'Issy le 4 décembre 1929», cité dans Divo, 2015: 78. 
Le principe est alors résolument celui de l'éducation populaire. La transmission verticale de connaissances est d'ailleurs récusée dans le manuel jociste: "surtout pas d'exposé théorique, pas de définition, pas de leçon de professeur à élèves, mais une conversation familière ${ }^{6}$. Prêtres et militants catholiques ont alors le sentiment que cette méthode inductive distingue la JOC-F des écoles ouvrières socialistes et communistes. "On voit par là la supériorité de la formation jociste sur le dogmatisme marxiste ", note ainsi L'Appel de la JOC (s.d.). Cet ouvrage constitue en quelque sorte un manuel de la JOC française. Il affirme que «les marxistes bourrent le crâne de leurs adeptes » (ibid.) alors que les militants ouvriers catholiques tireraient leurs connaissances et leurs conclusions des enquêtes qu'ils mènent. Pourtant, dès les années 1930, la relecture des faits et de l'influence donne une place importante aux aumôniers en tant que conseillers. Ils sont ceux qui transmettent les éléments de doctrine catholique. En 1938, une brochure à leur destination précise le rôle central des prêtres: "ne pas oublier qu'il y a autant d'occasions, sinon plus, de faire la formation chrétienne à l'occasion de la révision d'influence et de l'enquête, puisque les aumôniers les aident à juger ${ }^{7}$.» Le déchiffrement de faits qui montrent "l'influence" du mouvement constitue bien, sous le contrôle vigilant d'un prêtre, le cœur de la méthode jociste originelle.

Ces pratiques de l'Action catholique spécialisée puisent toutefois dans un répertoire de formes existantes, même si la mémoire en circulation ultérieurement dans les mouvements tendra à l'ignorer ou le minorer. La relecture peut être une déclinaison des exercices de retraites que pratiquaient les prêtres auprès d'ordres religieux spécialisés dans l'accompagnement spirituel ou qu'ils avaient connus au cours de leur formation au séminaire. L'Action catholique générale, organisée autour du "piété, étude, action » de l'Association catholique de la jeunesse française (ACJF), accordait également de l'importance à l'enquête qui permettait la formation de ses membres, même ouvriers ou employés. Avant l'ACJF, le cercle d'étude doit assurément beaucoup au mouvement du Sillon de Marc Sangnier. Comme l'a établi Marie-Emmanuelle Chessel dans une étude récente, "dans de nombreux mouvements organisés de laïcs catholiques, dès le début $\mathrm{du} \mathrm{xx}^{\mathrm{e}}$ siècle, la cellule de base apparaît être celle des “cercles d'études”. Ces groupes sont soumis à "l'animation spirituelle des aumôniers" et à la "présidence d'un laïc" "(Chessel et al., 2018: 162). Selon l'historienne, l'apport de l'Action catholique spécialisée dans l'entredeux-guerres vient surtout de l'adjonction d' "un nouveau travail d'enquête nationale articulée à une "révision de vie", c'est-à-dire à la relecture des faits à la lumière de l'Évangile» (ibid.).

En tout cas, dès l'origine la révision de vie paraît être, du moins à la JOC, davantage qu'un outil pédagogique ou pastoral (Dubreuil, 1999). Elle renvoie à des mutations spirituelles plus générales au sein du catholicisme français.

6. Manuel de la JOCF, 1929127.

7. L'Action de l'aumônier, ce que les dirigeants jocistes attendent de leurs aumôniers, 1938. 
Étudiant les théologies, qui sous-tendent les mouvements d'Action catholique spécialisée, Jean-Hugues Soret (2007) a pu être sensible à la façon dont la spécialisation du laïcat témoigne de la percée d'idées nouvelles dans la philosophie et la théologie françaises. Dans les années 1930, les aumôniers français enrichissent la méthode pédagogique belge avec un discours théologique, si ce n'est mystique, cherchant à spiritualiser le quotidien. Faut-il y voir l'assimilation de la philosophie de Maurice Blondel, le penseur d'une action dont le sens à déchiffrer ne peut pas précéder son accomplissement? Cela se traduit par une sensibilité accrue aux événements qui interviennent dans la vie du militant. Il convient de les déchiffrer au cours d'un moment militant spécifique qui se sépare progressivement du cercle d'étude type ACJF ou le Sillon. Le premier aumônier national de la JOC, Georges Guérin, le note lui-même:

La JOC a connu en 1936/37, non pas simplement la secousse de nouvelles lois sociales, mais il y a eu dans la JOC [...] cette vision qui est à l'origine de la Révision de vie. [Auparavant] on en était à la démarche inverse: on parlait à la JOC de révision d'influence. Les militants, autour d'une table, devaient dire quelles influences ils avaient pu avoir dans la semaine. Ce n'était pas bien facile. C'était décourageant. Voilà qu'un militant découvre les «richesses de la masse », il a été vraiment révolutionnaire: il ne faut pas de révision d'influence. Le Seigneur est présent dans le monde. Les copains sont autrement plus dociles au Seigneur que nous. Quand donc nous allons à la rencontre des autres hommes pour annoncer le message du Christ, la grâce du Christ nous a précédés ${ }^{8}$.

Il est difficile de considérer ce basculement au moment du Front Populaire comme un fait indiscutable. Il s'agit peut-être d'une illusion chronologique rétrospective du fondateur du jocisme français. Néanmoins, la naissance de la révision de vie au sein de la JOC s'énonce encore aujourd'hui sous ce motif narratif (Béjot, Gau, 1978). Aux frontières de la théologie et des sciences historiques, une étude de Jean Divo portant sur le mouvement dans le diocèse de Besançon tend toutefois à corroborer cette rupture chronologique à partir d'un exemple local (Divo, 2015). Plus largement, une reconfiguration des pratiques après le Front populaire paraît vraisemblable. Les occupations d'usines, les mobilisations sociales et les acquis qu'elles ont générés, ont été vécus par des militants comme des avancées favorables au mouvement ouvrier.

En effet, la JOC-F est née sur un programme de reconquête incarné par le slogan «nous referons chrétiens nos frères ». Issu d'une strophe de l'hymne jociste, il témoigne de l'intégralisme catholique des débuts des mouvements attachés alors à bâtir une «nouvelle chrétienté » ${ }^{9}$. Mais l'animation spirituelle du milieu puis un idéal missionnaire plus réaliste prennent assez rapidement le relais. Dans les années 1930-1940, les aumôniers, suivis bientôt par les militants, cherchent à donner un sens nouveau à ce qui a été entrepris.

8. Note manuscrite de l'abbé Guérin sur l'histoire de la JOC, s. d., Fonds Georges Guérin, Centre national des archives de l'Église de France, 53 CO 127.

9. Voir, par exemple, Cardijn, s. d. 
Effectivement, le résultat des efforts accomplis est encore limité par rapport à l'ambitieux programme initial de régénération chrétienne des milieux populaires. Mais, dans le quotidien de l'atelier, de la famille, du quartier, ou encore dans les mobilisations sociales du mouvement ouvrier, des signes sont à relever. Ils attestent du fait que quelque chose d'ordre chrétien est, malgré tout, en train de se produire et que les efforts militants déployés ont du sens. En proposant d'intégrer le quotidien dans une pratique à finalité religieuse, l'Action catholique peut alors mettre en œuvre une formule novatrice. La révision de vie procure au militant le sentiment qu'il n'est pas chrétien qu'à l'église, dans ses rites et ses œuvres, mais également dans sa vie personnelle et ses engagements militants propices à un monde plus juste et conforme à l'Évangile ainsi qu'à la volonté de Dieu. Cette pratique est l'une des réponses possibles pour maintenir un lien avec l'expérience personnelle et professionnelle des croyants. La révision de vie se stabilise donc avant la Seconde Guerre mondiale comme une pratique jociste qui se diffuse, peu à peu, bien au-delà des mouvements spécialisés.

\section{La révision de vie au cœur des " trente glorieuses" du militantisme d'Action catholique spécialisée (années 1940-années 1970)}

Des années 1940 aux années 1970, la révision de vie connaît une période phare. La pratique accompagne l'essor de l'Action catholique spécialisée au sein du laïcat français. Dans une configuration théologique particulière, de plus en plus marquée par l'attention portée aux "pierres d'attente » ou aux "signes des temps ${ }^{10}$ du père Chenu ${ }^{11}$, la révision de vie participe au grand moment militant du catholicisme de l'avant et après concile Vatican II. Mais comment expliquer un tel succès?

\section{Le contexte porteur de l'après-guerre}

Peu d'études font le lien explicite entre la percée de nouvelles thématiques théologiques et cet essor de nouvelles pratiques de l'après-guerre. L'historiographie est plus sensible aux condamnations intellectuelles des courants trop avancés (Fouilloux, 2006) ou à la mise à pied de l'expérience des prêtres ouvriers (Leprieur, 1989). Pourtant, la confrontation se joue aussi sur le terrain plus

10. La question des «signes des temps" trouverait son origine dans des travaux réalisés dans les années 1940-1950. Elle a été fortement mise en avant dans les textes importants de Vatican II comme l'encyclique Pacem in terris de Jean XXIII (1963) et surtout dans la constitution pastorale Gaudium et Spes (1965). Sur cette question, le théologien québécois Gilles Routhier (2012) offre un beau panorama de l'expression et de son évolution.

11. Le père Marie-Dominique Chenu (1885-1990) a développé une théologie qui articule l'histoire et le christianisme. Dans son œuvre, on retrouve plusieurs fois l'expression de "pierres d'attentes » pour désigner les réalités humaines ouvertes à la grâce. Voir Geffré, 1985. 
discret des outils pastoraux. La révision de vie reflète le besoin éprouvé par des militants laïcs de développer un outil leur permettant d'accroître leurs capacités d'agir dans la société, notamment dans le militantisme syndical, malgré les réserves répétées de leur institution d'appartenance (Tranvouez, 2000).

Étudiant la différence entre les moments «moderniste » et «progressiste » ${ }^{12}$, Thierry Keck note que le premier correspond à une "approche critique [...] visant une conception du dogme restreinte à un thomisme fermé », alors que le second conduit ses acteurs «sur le terrain social, à la jonction du monde de la pensée et de l'action, des intellectuels et des militants de la mission ouvrière » (Keck, 2013 : 254). En plaçant leur action sur le terrain de la spiritualité et de la pastorale, à travers la révision de vie, les acteurs de l'Action catholique ouvrière ont évité celui plus litigieux de la théologie dogmatique au moment où Rome condamnait. La révision valorise une spiritualité ancrée dans le quotidien, attachée à la résolution d'enjeux concrets, et met à distance une approche trop intellectuelle de la foi. La révision de vie n'enlève rien, du moins en apparence, au catholicisme paroissial traditionnel. Elle peut s'accomplir de manière peu suspecte dans une salle d'œuvre et sous la conduite d'un prêtre.

En dehors d'explications internes au catholicisme, nous pouvons également rattacher l'essor de la révision de vie au contexte plus général des mutations de la société française. Bien qu'il s'agisse du cadre québécois, Michel Beaudin a ainsi suggéré l'idée selon laquelle l'Action catholique du Canada français était bien adaptée aux structures socio-économiques de son époque. Sa réflexion serait recevable pour le contexte français. L'organisation pastorale qui promouvait la révision de vie fonctionne bien dans le "modèle du capitalisme à visage humain connu sous le nom de fordo-keynésianisme (1935-1970) » (Beaudin, 1996: 75). Le déchiffrement d'un sens historique et religieux des événements peut fonctionner lorsque les conditions de vie s'améliorent et que, à la source des conquêtes sociales, on trouve des engagements militants et des mobilisations collectives. La révision de vie accompagne ainsi l'irrépressible besoin de "prise de parole» des années 1968 (Certeau, 1994). Elle nourrit un désir de s'exprimer individuellement, ici dans un espace semi-public, sur des problèmes tant individuels que sociaux, dans le but de changer la vie.

\section{Le « fait de vie » : base et aboutissement de la révision}

Si la pratique est faiblement repérée dans l'historiographie, qui est plus sensible aux débats d'idées et aux condamnations intellectuelles, la matière archivistique

12. Le «modernisme» désigne un courant intellectuel catholique, regroupant des travaux variés condamnés en bloc par Rome au début du $\mathrm{xx}^{\mathrm{e}}$ siècle. Ces derniers, contrairement au néothomisme enseigné dans les universités et séminaires catholiques, commencent à employer des outils historico-critiques dans l'approche des textes sacrés et à historiciser la formulation des dogmes (Colin, 1997). La crise progressiste désigne, quant à elle, un moment de l'après-guerre et de mise en place de la guerre froide durant lequel les solidarités militantes et intellectuelles nouées entre catholiques et communistes dans l'expérience de la Résistance et de la Libération ont été condamnées par la papauté. 
produite par la révision de vie est en quantité déroutante pour l'historien. Elle se caractérise par son abondance et sa variété (carnets personnels, comptes rendus de révisions réalisées en groupe, articles permettant de démarrer la révision). Le statut des documents est pourtant difficile à déterminer: sont-ce des "archives de soi », de la littérature grise militante ou un mélange des deux?

Dans des mouvements comme la JOC et la JOCF, tous les supports militants, les périodiques, mais également les rapports d'activité ou d'orientation sont organisés autour du triptyque «voir, juger, agir ». Ils reposent sur l'exploitation de «faits de vie » qui sont la base de la réflexion collective. Ces petites scènes vécues, racontées au cours des révisions de vie, sont collectées, à l'origine, par les militants et les responsables. Résultats des questionnaires diffusés pour la réalisation de l' «enquête-campagne », extraits des carnets de militants rapportés en réunion, retranscriptions de témoignages réalisées devant une équipe, les multiples «faits de vie» circulent au sein des mouvements. Ces séquences prélevées au cours des partages sont réinjectées dans des articles de presse afin qu'elles servent de base de réflexion. Si un fait est remarqué, il peut même être demandé à un militant ou à un groupe de rédiger une "monographie ", version plus détaillée et plus précise. La pratique de la révision de vie nourrit ainsi à la JOC toute une pédagogie par l'exemple. Les faits de vie peuvent être vus comme de petites paraboles signifiantes. Ils incarnent les valeurs à cultiver en tant que bon militant. Les faits évitent d'exprimer de manière trop doctrinale et intellectuelle ce qui est attendu dans l'action. Au conseil national de la JOCF de 1955, ce fait issu d'une révision est présenté au sein même du rapport d'orientation:

Lulu est au centre, le professeur leur donne une composition française sur les Femmes savantes de Molière avec ce thème "Que penser de l'affirmation: "Il n'est pas bien honnête, et pour beaucoup de causes, qu'une femme étudie et sache tant de choses" ». Dans son devoir, Lulu a donné la priorité aux valeurs féminines dans l'éducation de la femme, d'où la réaction du professeur qui lui dit qu'elle n'a pas d'idéal et qu'elle espère que ses élèves rêvent à autre chose qu'à repriser les chaussettes de leurs maris et à être de vulgaires bonnes! Elle, qui est mariée, fait la classe, donne des cours supplémentaires, trouve le temps de se cultiver par de la lecture et, pour autant, n'abandonne pas son foyer, elle a une fille élevée, par une vulgaire bonne d'ailleurs. Très forte réaction de Lulu qui s'est faite traiter d'insolente, mais a repris la discussion avec les filles dans la cour de récréation.

Le fait de vie a été retravaillé ici par la permanente qui le transpose en une anecdote énoncée à la troisième personne. Elle établit un antagonisme fort et didactique entre "Lulu ", qui défend les "valeurs féminines ", et sa professeure. Cette dernière semble faire du travail salarié et de la culture les seuls moyens de l'émancipation féminine. La professeure est, en quelque sorte, dénoncée par «Lulu » comme porteuse d'une violence sociale. Cette dernière est engagée dans un mouvement, qui défend alors l'amélioration statutaire du travail domestique, notamment par la recherche de conventions collectives pour les employées de maison. Lulu ne comprend donc pas pourquoi il est 
reproché à quelqu'un dans cette condition d'être dominé et de manquer d'ambition. À ses yeux, la professeure méprise le travail domestique et oublie que sa propre émancipation passe par le travail salarié d'une "vulgaire bonne». Comme il est donné aux différentes déléguées fédérales, ce fait de vie peut également être considéré comme une forme de partage d'une ligne jociste. Une émancipation, qui passerait par la mise à l'écart du travail domestique et de la sphère traditionnelle féminine, en accordant beaucoup d'importance aux études, reste encore récusée au milieu des années 1950. La révision de vie est aussi, comme ici, un puissant outil de mise en narration de l'expérience individuelle au service d'un groupe et de son idéologie.

\section{La révision de vie, outil de formation militante}

Étudiant les révisions de vie, on relève vite un écart entre le but de l'exercice (l'introspection personnelle, le discernement individuel) et l'aspect finalement assez stéréotypé des réponses. Comment un temps réflexif et personnel peut-il générer des documents assez normatifs? La sociologue Françoise Richou a pu s'essayer, dans ses travaux sur la JOCF, à cerner l' "habitus » des militants jocistes. Travaillant le concept sociologique, elle s'en est servi pour désigner les dispositions acquises des jocistes pour le combat militant. Ses travaux montrent comment la JOCF et la JOC peuvent aussi être appréhendées comme des mouvements chrétiens d'éducation populaire au profil sociologique très marqué (Richou, 1997). Elle a toutefois été moins sensible à la façon dont la révision de vie s'inscrit au cœur de cet apprentissage militant. L'étude des pratiques militantes autobiographiques en usage au sein du Parti communiste aurait pourtant pu offrir un point de comparaison intéressant. Dans l'introduction d'un ouvrage paru en 2002, Claude Pennetier et Bernard Pudal exposent l'historiographie actuellement dominante des autocritiques marxistes. Cette dernière interprète ces pratiques, énoncées à la première personne et réalisées en groupe (à l'instar des révisions de vie), comme une forme de gestion des populations, de sélection, de promotion et de contrôle des nouvelles élites sociales que sont les cadres du mouvement (Pennetier et Pudal, 2002). Cette voie est pourtant encore peu envisagée dans l'historiographie de l'Action catholique.

À la JOC et la JOCF, la révision de vie constitue pourtant un espace de parole à la première personne sous contrôle. L'autonomie individuelle consentie au réviseur adulte ou juvénile est contrebalancée par un imposant dispositif normatif de production du fait de vie. En amont de l'enquête, de la documentation est ainsi rassemblée. Des prêtres peuvent fournir des "éléments de jugement» (conférences, topos). Il existe également, dans la presse des mouvements JOC et JOCF, des grilles qui aident les militants à organiser les révisions de vie. Elles sont produites par les permanents salariés des mouvements et diffusées dans les périodiques pour affûter le regard des jocistes. Ces grilles proposent d'identifier les faits à partir des situations concrètes 


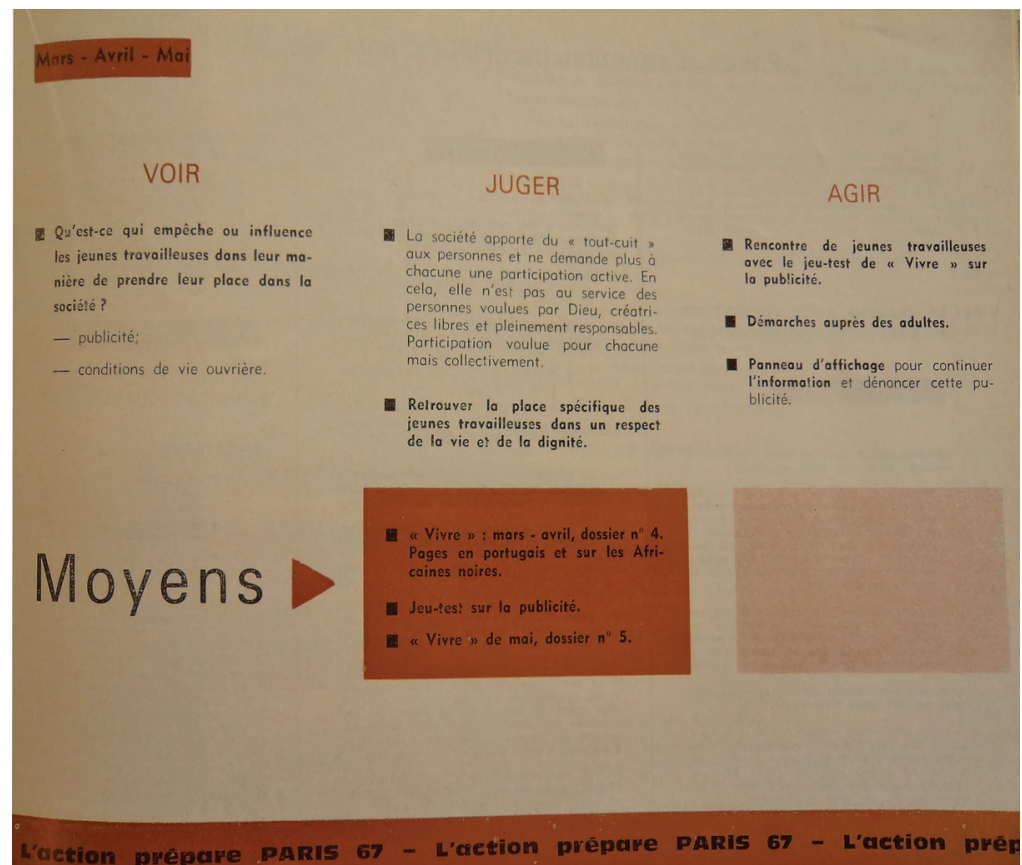

que connaissent les jeunes à l'école, à l'usine ou au bureau, et de donner des pistes d'interprétation.

Fig. 1: Extrait du carnet de préparation du rassemblement «Paris 67", JOC et JOCF, Archives départementales des Hauts-de-Seine, $45 \mathrm{~J}$.

Dans le jocisme, il existe donc un dispositif militant assez sophistiqué pour éduquer les militants à bien saisir des faits, à les relever et, au-delà de ce premier travail, à les interpréter. Aux Archives départementales des Hauts-de-Seine, dans le fonds $45 \mathrm{~J}$ qui conserve les dépôts du secrétariat national JOCF sur la période 1928-1987, on trouve des petites boîtes métalliques. Ces dernières répertorient, par un système d'attaches de couleur, des notices abrégées des faits de vie et monographies conservées au niveau national. L'existence de ce fichier reflète la façon dont sont produites les grilles cherchant à préparer les révisions de vie.

Lorsqu'une permanente produisait un support militant (rapport, article, tract) concernant un thème, un groupe d'âge ou d'activité en particulier, elle identifiait les faits de vie et les monographies déjà existants, à l'aide de cet index. Les textes servant de supports nationaux sont rédigés comme une « révision de vie » générale du mouvement sur différents problèmes sociaux. Ils sont, à la fois, la somme des expériences collectées à la base et une unification de leur mise en forme. Il est difficile de dire s'il s'agit d'une pratique isolée à la JOCF ou si elle est répandue, plus généralement, dans l'univers du 
IO - ARChIVES DE SCIENCES SOCIALES DES RELIGIONS

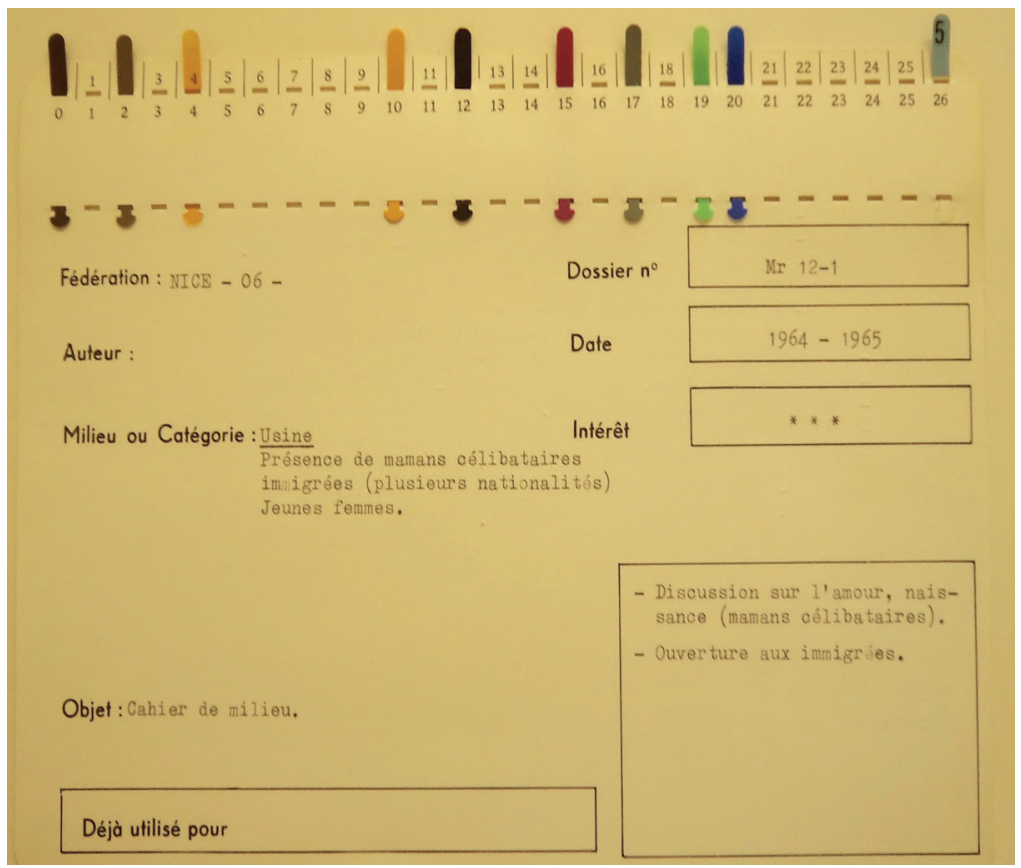

Fig. 2 : Fiche d'analyse d'un fait de vie, JOCF, 1964 ou 1965, Archives départementales des Hauts-de-Seine.

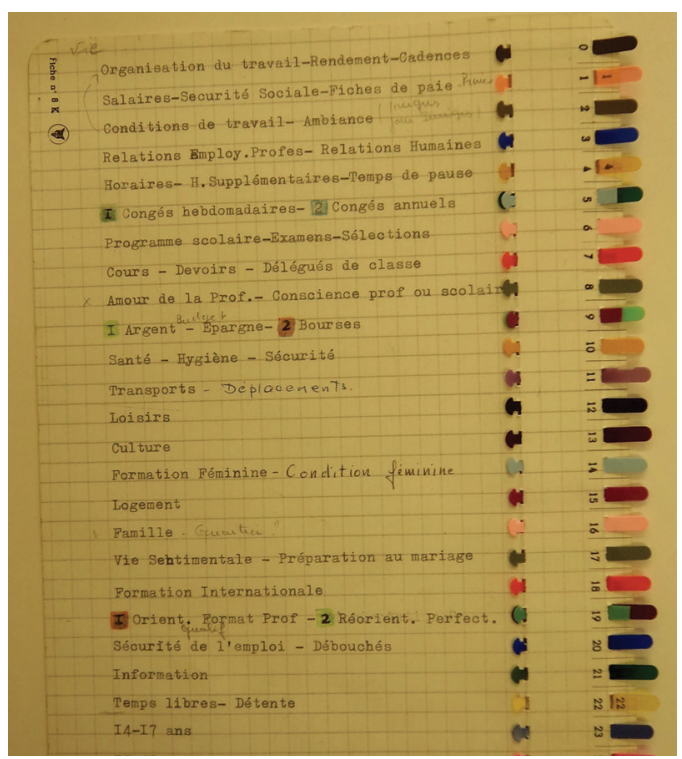

Fig. 3 : Légende des codes couleurs. 
militantisme d'Action catholique spécialisée durant les années conciliaires pourtant si porteuses.

\section{Une pratique consacrée au moment du concile Vatican II?}

En 1967, la Conférence des évêques de France exprime son attachement à l'Action catholique spécialisée. Elle «donne le ton, le sens de la marche, le style de la présence " ${ }^{13}$. L'épiscopat encourage donc la pratique de la révision de vie dans le contexte de l'aggiornamento pastoral entrepris depuis l'ouverture du concile Vatican II en 1962. En réalité, les textes qui en sont issus en parlent peu. Contrairement à certaines espérances françaises, les pères conciliaires ne font de l'Action catholique spécialisée qu'une des formes possibles d'organisation du laïcat catholique ${ }^{14}$. Vatican II étend toutefois la pratique de la révision de vie à des cercles bien plus larges. Les prêtres et les religieux sont invités à l'effectuer, s'ils le souhaitent, dans le cadre de la rénovation de leur règle. Expression bien dans son époque, dans son message de clôture adressé aux jeunes, Paul VI présente le concile lui-même comme une «imposante révision de vie ${ }^{15}$.

En France, l'exercice devient surtout un objet de réflexion théologique dans un milieu plus large que celui de la pastorale ouvrière. Paul Airiau, prêtre engagé dans l'Action catholique, publie en 1960 Agir en vérité. En 1964, le dominicain Jourdain Bonduelle édite une série d'articles et de conférences sous le titre La Révision de vie, situation actuelle. L'essai fournit une étude approfondie sur l'histoire et l'avenir de la pratique. Pourtant, ces articles et ouvrages de théologie ou de pastorale n'expliquent que secondairement dans quelle mesure elle est alors répandue en France. Si l'Action catholique des milieux indépendants (ACI), l'ACO et les différentes jeunesses catholiques pratiquent l'exercice, avec des approches variables que l'on connaît encore mal, on ne la retrouve pas dans des lieux d'engagement du laïcat aussi divers que les équipes enseignantes, les équipes Notre-Dame ou dans les groupes «Vie nouvelle », comme le note le dominicain Bonduelle. Le scoutisme lui préfère généralement le «temps spi(rituel) », qui n’obéit pas forcément au principe de la relecture biographique. Néanmoins, le modèle possède une certaine attractivité comme le révèle, par exemple, l'étude de Marie-Emmanuelle Chessel. Le Centre français du patronat chrétien, héritier de la Confédération

13. Assemblée plénière de l'épiscopat français, «Déclaration, orientations sur les exigences missionnaires ", Documentation catholique, 64, 1967, col. 2036-2037.

14. L'édition bilingue et révisée des textes conciliaires (Concile du Vatican, 2002) place dans son index deux entrées "révision de vie » : l'une concerne le paragraphe 30 du décret sur l'apostolat des laïcs, qui la présente en des termes très larges et dans une perspective très missionnaire ; l'autre concerne le paragraphe 14 du décret sur le ministère et la vie des prêtres qui conseille, en plus des exercices de piété, "la vérification concrète" de la qualité de vie sacerdotale "afin de discerner quelle est la volonté de Dieu».

15. Paul VI, «Aux jeunes », Messages du Concile (8 décembre 1965), in Concile du Vatican, 2002. 
française des professions d'entre-deux-guerres, a eu pendant longtemps un mode organisationnel proche d'un syndicat. En 1955, sous l'action des conseillers spirituels, son bureau national suggère d'implanter «la méthode ACI ». Elle repose sur une méditation personnelle réalisée sur un texte d'évangile se rapportant à un sujet annuel avant une mise en commun de ces réflexions (Chessel et al., 2018: 168).

Selon les indications chiffrées de l'épiscopat français de 1960, 125000 jeunes catholiques, au sein de la JEC-F, de la JAC-F et de la JOC-F, pratiqueraient potentiellement la révision de vie ou des variantes plus ponctuelles. L'ACI et l'ACO ajoutent quelques milliers de militants adultes à ce noyau de «réviseurs" juvéniles ${ }^{16}$. La révision de vie reçoit peut-être un écho disproportionné par rapport à son importance réelle. Cependant, il convient de préciser que, à l'image de l'Action catholique, elle se trouve au cœur des enjeux idéologiques de la réception du concile Vatican II en France. Dans les milieux optimistes quant aux changements entrepris, la pratique devient l'emblème d'un nouveau laïcat, engagé et autonome. Dans une conférence restée célèbre sur «l'avenir de l'Église ", prononcée par le dominicain Yves-Marie-Joseph Congar lors de la Semaine des intellectuels catholiques de 1963, la révision de vie est présentée comme «le fruit et le signe de la reconstitution d'un homme chrétien» (Congar, 1964: 211-212). En cherchant Dieu dans tous les aspects de leur vie, y compris les plus triviaux, les chrétiens pratiquant la révision de vie « vivent les exigences du christianisme moins à partir de règles homologuées une fois pour toutes par l'Église, extérieures à leur propre conscience, mais à partir de ce que la vie, les événements, les circonstances leur révèlent comme exigence ou appel à la lumière de l'Évangile» (ibid.).

\section{La révision de vie, élément central ou secondaire de la "crise catholique"?}

Au cœur des pratiques militantes, au moment des années de réception du concile Vatican II en France, la révision de vie est un élément méconnu, pourtant central, de la "crise catholique » de l'après-concile (Pelletier, 2002). Que devient une pratique, conçue originellement comme une pédagogie chrétienne d'approfondissement spirituel, dans un contexte de forte politisation des engagements et de contestation des formes religieuses?

\section{Les rendements décroissants du motif des «signes des temps »}

Avant même l'entrée en crise du militantisme catholique, des doutes avaient pu être formulés quant à l'optimisme missionnaire dont on auréolait la révision de vie et, derrière elle, l'Action catholique. Dans Le Supplément à la vie

16. Rapport confidentiel de s. Exc. Mgr Renard à l'Assemblée plénière de l'épiscopat français, avril 1960, p. 30-31, Archives Historiques de l'Archevêché de Paris (AHAP) : 1 B 2 2a. 
spirituelle de 1964, un dominicain, Jean-Pierre Jossua, se demande le premier "où en est la théologie de la "révision de vie" et de "l'événement" ? (Jossua, 1964). Cet article cherche à situer l'exercice dans les courants montants de la théologie de l'époque. Il rattache la pratique aux nombreuses théologies de «l'événement», des «réalités terrestres» ou bien de «l'incarnation ». Son auteur n'entretient alors pas de contact avec l'Action catholique ${ }^{17}$. Cette liberté lui permet d'évaluer positivement ce qu'apporte la pratique, mais également de formuler quelques questions sur son devenir. Elle tend, selon lui, à retrouver l'eschatologie des premiers siècles en survalorisant la promesse de l'avènement d'un nouveau type de société, risque dont étaient bien conscients, semble-t-il, les théologiens travaillant l'expression de "signes des temps », dans le schéma conciliaire qui allait devenir la consistution pastorale Gaudium et spes (Routhier, 2012).

Cette critique, somme toute très mesurée, du dominicain Jossua précède celle plus cinglante et retentissante de Jean Lebrun dans Esprit en 1979. Le déchiffrement des "signes des temps " ne conduit-il pas les militants à trop vite confondre des choix antérieurement faits par idéologie et les événements tels qu'ils sont relus? (Lebrun, 1979). Il faut dire qu'entre-temps, des tensions entre mouvements d'Action catholique et évêques français avaient surgi dans les années 1965-1975 à travers une série de crises (Prudhomme, 2012). L'exercice de révision ne résiste pas à la critique plus générale portée par le courant structuraliste. Les enquêtes sont autant des outils de description du monde que de production des représentations, si ce n'est de discipline des comportements. La période devient celle d'un questionnement sur l'identité chrétienne des mouvements et de la pratique qui supporte leurs engagements.

En 1970, la JEC abandonne ainsi la révision de vie pour une lecture matérialiste de la Bible. Cette dernière est la transposition à l'exégèse de la grille de lecture marxiste adoptée pour la conduite nationale (Giroux, 2013: 517 sq.). D'autres causes plus structurelles peuvent être avancées dans la crise de la pratique. Avec la baisse des vocations sacerdotales, les prêtres dévolus à l'accompagnement spirituel des mouvements sont moins nombreux. Conçue pour des jeunes de milieux populaires, certainement peu pratiquants, mais disposant d'un "acquis chrétien ", la révision de vie perd aussi de son efficacité, comme le note le théologien Xavier Dubreuil: «il n'est plus guère possible de prendre appui sur une mémoire chrétienne pour appeler à l'agir, ni de faire comme si l'expérience de l'action, plus ou moins confrontée à l'Évangile, pouvait facilement se vivre comme une réelle expérience de foi » (Dubreuil, 1999: 54). En novembre 1976, dans le cadre d'évolutions plus générales liées aux orientations prises par la JOC européenne, la JOCF distingue même la révision de vie "ouvrière" de la révision de vie «de foi » (Orieux, 1998). La première est spécifiquement consacrée à l'évaluation concrète de l'engagement militant, la seconde est ouverte aux militantes qui souhaitent approfondir une

17. Entretien réalisé le 24 novembre 2016. 
démarche de foi. L'analyse des structures de domination se substitue ainsi à la pédagogie initiale de la révision de vie. La grille de lecture «macro-économique » l'emporte sur le travail du sujet engagé dans le déchiffrement de son quotidien. La percée d'un "marxisme chrétien », substitut du messianisme évangélique, disqualifie un exercice comme la révision de vie, qui appelle plutôt à la relecture personnelle.

\section{La révision de vie comme outil de sécularisation}

La pratique a-t-elle pu finalement jouer un rôle dans la subversion des normes élaborées par l'institution catholique? Ce phénomène est surtout repéré chez les clercs. Étudiant les changements liés au concile Vatican II dans la Province de France des frères dominicains, Yann Raison du Cleuziou voit ainsi dans l'apparition de la révision de vie au couvent d'étude des frères, à la Tourette (Éveux) près de Lyon, une évolution majeure de la vie religieuse des années 1960. Elle accompagne les changements au sein de l'enseignement en substituant au respect de la règle en soi, de moins en moins acceptée par les frères étudiants, le critère d'évaluation plus large de "l'Évangile ». Pour l'historien, cette révision de vie correspond à "l'infrastructure théologique " dont le catholicisme français avait besoin après-guerre pour satisfaire in fine le programme modernisateur porté par les jeunes générations de religieux: «La révision de vie [transforme] en profondeur l'économie des grandeurs religieuses qui configurent la vie dominicaine dans les années 1960: les évangiles sont substitués à la règle comme norme de référence pour évaluer l'authenticité religieuse des conduites» (Raison du Cleuziou, 2012:38).

Ainsi, la pratique de la révision a pu avoir un potentiel sécularisateur dans la mesure où elle permet de réévaluer à une aune nouvelle les nombreuses pratiques de vie religieuse héritées pour les remettre en cause. Il n'est donc pas étonnant que l'institution cherche à reprendre la main sur une méthode aussi déstabilisante. En France, la première étape de cette reconquête des mouvements d'Action catholique par l'épiscopat date de 1975. Pour ne pas les compromettre dans leurs combats politiques, les évêques français mettent fin au mandat au nom duquel les militants agissent, sans pour autant supprimer les mouvements. Les «militants » sont appelés à se rapprocher de l'offre ordinaire des sacrements dans les paroisses. L'hégémonie pastorale de l'Action catholique spécialisée est également concurrencée par l'essor de nouveaux mouvements de laïcs. Les évêques proposent, avec la bénédiction des autorités romaines, d'autres formes d'engagement social, de pratiques et de dévotions.

Toujours en 1975, Paul VI reconnaît ainsi officiellement le «Renouveau charismatique» catholique, né sur les campus nord-américains au contact des mouvements du réveil américain, pentecôtistes ou autres. Les nouveaux venus placent au cœur de leur foi l'effusion spirituelle que procurent les chants, les prières ou des sacrements esthétiquement présentés, davantage que les groupes de parole et la recherche réflexive. L'interprétation théologique qui tend à s'imposer dans l'au-delà de la crise catholique insiste plutôt sur la vocation de 
chaque laïc à parvenir à la sainteté dans les formes instituées de l'Église avant d'aller la chercher dans les espaces de mission. Lors du voyage en 1980 de Jean-Paul II en France, le pape ne manque d'ailleurs pas de rappeler que «la révision de vie peut gagner en précision et l'action en efficacité profonde ${ }^{18}$. Le réarmement progressif des vieilles formes sacramentelles ou de piété, chez une minorité de croyants, que l'on ne qualifie pas encore "d'identité " (Dubreuil, 1999: 54) mais qui en ont les attributs, discrédite peut-être des pratiques comme la révision de vie.

\section{La révision comme lieu du «bricolage » catholique}

Il n'est toutefois pas dit que cette dernière puisse expliquer l'affaiblissement de la pratique catholique. Dans son étude sur la confession dans la France contemporaine, Guillaume Cuchet ne voit d'ailleurs pas de concordance nette entre le moment d'apogée de la révision de vie et la chute brutale de la confession: "dans la littérature assez abondante consacrée au courant des années 1950-1960, à la question de la "révision de vie" au sein de l'Action catholique et au-delà, il n'était pas question de chute parallèle de la confession, point qu'on n'aurait pas manqué de signaler le cas échéant, dans la mesure où l'un des enjeux doctrinaux était bien de distinguer les deux " (Cuchet, 2015 : 407). De manière courante, on associe les processus de sécularisation aux questions de genre et d'intime. L'affaiblissement des grands systèmes sociaux de croyances et de pratiques passerait, de manière non négligeable en Occident, par la contestation des normes qui s'appliquent sur les corps et les sexualités, notamment au moment du «tournant subjectif » des années 1960 (Brown, 2009: 304; Turina, 2012: 199 sq.). La révision de vie est ainsi un bon observatoire de l'évolution des normes de genre catholique et de la façon dont elles se transmettent. Les groupes qui la pratiquent ont ainsi connu à la JOC une évolution riche de sens au cours des années 1960-1970. Ils peuvent devenir localement des groupes de parole mixtes. Face à la demande grandissante de certains membres de militer "en couple», des groupes de fiancés ont été adjoints aux groupes de révision de vie (non mixtes). On y aborde, toujours selon la méthode de la relecture biographique, les problèmes spécifiques de ceux et celles qui se préparent au mariage, par exemple ici, dans une révision de vie de couple. Elle date de 1977, à un moment où la "crise catholique » avait largement commencé:

Sylvie [et Patrick]: Mes parents ne s'entendaient pas; ils ont divorcé à 10 ans. Je suis l'aînée de 5 enfants. J'ai gardé depuis l'âge de 7-8 ans mes sœurs. Je manquais tous les samedis l'école. Je travaille depuis 4 ans à l'hôpital. J'ai fait des stages avant pour avoir le CAP collectivité. Mon information sexuelle, je l'ai eue à l'école. Je

18. Discours de Jean Paul II aux responsables des mouvements de laïcs, 31 mai 1980, https:// w2.vatican.va/content/john-paul-ii/fr/speeches/1980/may/documents/hf_jp-ii_spe_19800531_apostolato-laici-francia.html (consulté le 27 mars 2019). 
ne prends pas de moyens contraceptifs. On ne prend pas de moyens contraceptifs. C'est moi qui décide. Les enfants qui viennent, on doit les garder. Nous, on croit en Dieu et vous, que pensez-vous de l'avortement?

Philippe: Par rapport à l'avortement, la meilleure solution: la contraception.

Maryse: Et si un enfant arrive?

Patrick: Pas de problème, dans la famille, en cas de coup dur, on s'aide. Mon père était routier ferrailleur, ma mère vendait des fleurs. On était trois garçons et deux filles. Mes parents s'entendaient bien. À 14 ans, j'ai été apprenti-jockey, après j’ai été en usine, puis j'ai été militaire de carrière pendant deux ans et demi. J'ai eu un accident, j'ai été réformé puis j'ai connu Sylvie. J'ai tout appris tout seul. On a dit que la pilule était dangereuse, alors, je ne veux pas qu'elle la prenne ${ }^{19}$.

À un premier niveau d'analyse, la JOC-F peut substituer ici à l'imposition d'une norme verticale sur les critères un échange sur un mode plus horizontal, en faisant advenir ce qu'on appellerait en termes laïcs un "groupe de parole» bien éloigné de la révision de vie originelle. Les problèmes éthiques sont résolus de manière discursive, sans forcément l'assistance d'un prêtre, à travers l'échange d'expériences entre pairs. La révision décentre le catholicisme de lui-même dans l'élaboration du jugement social et moral, en intégrant, par exemple, des savoirs exogènes (ce que disent les médias, l'école ou la science) dans l'analyse du réel. L'écoute entraîne le "non-jugement » du comportement d'autrui et décharge moralement les comportements autrefois combattus. Elle participe donc à la logique plus large de la sortie de «la pénitence». À un second niveau, toutefois, l'exemple donné révèle encore le relatif conformisme des deux couples de militants. Ils récusent les moyens contraceptifs et accueillent les enfants non choisis s'ils ne fonctionnaient pas. Les normes que l'institution seule ne parvient plus à transmettre sont comme re-légitimées par le groupe et son travail de délibération morale. La révision de vie peut ainsi être vue comme un dispositif de validation communautaire de normes religieuses, notamment celles de morale sexuelle, que le travail pénitentiel descendant ne parvient justement plus à faire vivre. En ce sens, elle participe plus au «bricolage» propre au religieux contemporain qu'à sa disparition.

\section{Le devenir de la révision de vie dans un contexte d'évolution spirituelle (des années 1980 à nos jours)}

Passée cette période de crise, la JOC-F des années 1980 reprend, aux côtés de l'ACO, la réflexion sur sa dimension missionnaire et le sens qu'elle accorde à la révision de vie. Cette dernière n'apparaît plus comme la panacée pour l'ensemble du catholicisme français, sans pour autant être disqualifiée. Un colloque à l'Institut catholique de Paris aboutit, au début des années 2000, à l'ouvrage Croire, vivre, raconter: la révision de vie (Pizivin et Strasser, 2003).

19. Fédération JOC Paris Sud, "Compte rendu de la rencontre jeunes couples ", 13 décembre 1977, Archives historiques de l'archevêché de Paris (AHAP) : 91 K 1, 31. 
Son sous-titre se veut un appel: «une pratique à réinventer». Dans son introduction, Daniel Pizivin, prêtre de la mission de France, veut voir dans la révision de vie une pratique porteuse. Elle s'insère dans le programme tracé par le rapport rédigé par Claude Dagens sous la forme d'une synthèse des travaux de ses collègues évêques. La révision répondrait ainsi particulièrement à l'objectif de "proposer la foi dans la société actuelle» (Conférence des Évêques de France, 1994-1996). La pratique lui paraît donc pertinente à condition de la réarticuler, toutefois, autour "d'autres pratiques ecclésiales » (Pizivin et Strasser, 2003: 38). Si l'institution consent à reconnaître ses vertus, elle demande aux mouvements de se penser également comme «catéchétiques » et liés à la transmission de la foi. Le regain d'intérêt annoncé est toutefois peu repérable au sein du catholicisme français très contemporain. Aujourd'hui, le mot n'apparaît pas dans le glossaire en ligne du site de l'Église catholique géré par les Évêques de France, alors que l'on y trouve, par exemple, une entrée "récollection ${ }^{20}$. Sur ce site, un document rédigé par un membre de l'ACO et un membre d'une équipe Vie chrétienne est toutefois en ligne. Datant de 2009, il «patrimonialise» d'une certaine manière la révision de vie, dans la mesure où le document la présente comme « un bien de l'Action catholique » légué à tous les croyants (Roche, Maincent, 2009).

Faut-il lier le déclin de la pratique tant à la reconfiguration pastorale des mouvements de laïcs par les autorités catholiques qu'au changement de contexte socio-économique et socio-culturel ? Toujours selon Michel Beaudin, l'Action catholique spécialisée répondait aux «défis de son époque, contrairement à la période post Trente Glorieuses marquée par la précarité ». La crise de l'État-providence, à partir des années 1980, «amplifie l'appauvrissement et la dislocation sociale ». À terme, il entraîne "un sentiment de fatalisme extrêmement démobilisateur devant le cours des choses et promeut ainsi un individualisme exacerbé» (Beaudin, 1996: 75). Lorsque le programme d'amélioration sociale s'essouffle, qu'il est en partie atteint ou qu'aller plus loin devient plus complexe, le récit de l'émancipation collective ne mobilise plus autant. Alors que le salut devient plus individuel que collectif, la relecture d'engagements militants et syndicaux est moins à l'ordre du jour, car elle est beaucoup moins fructueuse. Lorsque le déchiffrement des enjeux sociaux offre moins de rendements, une place plus grande est laissée au développement personnel et à la réalisation de soi. Les groupes de parole peuvent alors se substituer aux révisions de vie militantes dans des sociétés très sécularisées. Pratique encore vivante, normalisée au point d'être invisibilisée ou tombant progressivement en désuétude, la révision de vie semble donc avoir connu une forme d'acmé, avant rémission, dans l'au-delà des années 1980.

20. http://www.eglise.catholique.fr/glossaire/lettre/r/ (consulté le 23 avril 2019). 


\section{Conclusion}

La révision de vie est un élément central du catholicisme français du $\mathrm{Xx}^{\mathrm{e}}$ siècle. Mais elle fait aujourd'hui l'objet d'un intérêt historiographique extrêmement limité. La pratique a pourtant incarné une expérience pastorale originale, fondée sur la mise en récit du quotidien, au moment où la paroisse d'œuvres traditionnelle s'essoufflait et se détachait d'une société française qui s'industrialisait et se technicisait. Avant et après-guerre, la pratique accompagne même l'essor des mouvements d'Action catholique spécialisée alors qu'ils incarnent une avant-garde missionnaire que le Concile Vatican II révèlera dans toute son ampleur. Prise dans la crise catholique à l'instar des mouvements qui la propagent, la révision de vie connaît toutefois un recul, si ce n'est une récession après les années 1970 . Au fur et à mesure que des courants plus identitaires du catholicisme se développent, cette pratique religieuse s'est trouvée à son tour dépassée par des mouvements qui prônent le retour à une pastorale plus esthétique, attachée à la primauté de la liturgie, réservée sur les engagements collectifs et plus encline à faire vivre aux individus des expériences charismatiques et réparatrices. Observatoire des changements sociaux et religieux de la société française, et sûrement européenne, la révision de vie a laissé d'abondantes archives ainsi que des fonds documentaires qui sont souvent peu ou sous-exploités. Peut-on alors espérer qu'un nouvel intérêt naisse pour ces documents qui aujourd'hui tombent dans un relatif oubli? Il en va de notre compréhension de ce qu'était l'expérience religieuse au $\mathrm{XX}^{\mathrm{e}}$ siècle et des mutations qui ont affecté le catholicisme français.

\section{Anthony FAVIER \\ Laboratoire de recherche historique Rhône-Alpes (LARHRA, UMR 5190) anthony.favier@live.com}

\section{Bibliographie}

Airiau Paul, 1960, Agir en vérité, Paris, Éditions ouvrières.

Beaudin Michel, 1996, "Les Mouvements d'Action catholique comme pratique et théologie critiques", Laval théologique et philosophique, 52, 1, p. 67-84.

BÉJot Georges, Gau Étienne, 1978, Un Évêque à l'école de la JOC, Paris, Éditions ouvrières. Bonduelle Jourdain, 1964, La Révision de vie, situation actuelle, Paris, Éditions du Cerf. Brown Callum, 2009 [2001], The Death of Christian Britain: Understanding Secularisation, 1800-2000, Londres, New York, Routledge.

CARDijn Joseph, s. d., La Vie, le milieu, la masse, les trois pierres de touche de la JOC, leçons données aux journées sacerdotales de Vienne les 3 et 4 janvier 1937, "Réflexions jocistes ", Paris, Librairie de la jeunesse ouvrière.

Certeau Michel de, 1994 [1968], La prise de paroles, et autres écrits politiques, Paris, Éditions du Seuil.

Chessel Marie-Emmanuelle et al., 2018, L'Entreprise et l'Évangile. Une histoire des patrons chrétiens, Paris, Presses de Sciences Po. 
Cholvy Gérard, 2000, "Aux origines de la JOC française: le temps des conquêtes (1927-1943) », Revue d'histoire ecclésiastique, 95, 1, p. 215-242.

Colin Pierre, 1997, L'Audace et le soupçon. La crise du modernisme dans le catholicisme français, 1893-1904, Paris, Desclée de Brouwer.

Concile du Vatican, 2002, Vatican II. L'intégrale, Paris, Bayard.

Congar Yves M.-J., 1964, «L'Avenir de l'Église», L'Avenir, semaine des intellectuels catholiques, 6-11 novembre 1963, Paris, Fayard.

Cuchet Guillaume, 2015, «La crise du sacrement de pénitence dans le catholicisme français des années 1960-1970", Revue de l'histoire des religions, 23, 3, p. 397-428.

Divo Jean, 2015, L'Aubier, la JOC et la JOCF dans le diocèse de Besançon: 1927-1978, Paris, Éditions du Cerf.

Dubreuil Xavier, 1999, «Évolution du rapport Action/Foi au sein de la JOC», Cahiers de l'Atelier, 484, p. 50-57.

Foucault Michel, 2018, Histoire de la sexualité 4. Les Aveux de la chair, Paris, Gallimard.

Fouilloux Étienne, 2006 [1988], Une Église en quête de liberté: la pensée catholique française entre modernisme et Vatican II, 1914-1962, Paris, Desclée de Brouwer.

Geffré Claude, 1985, "Le Réalisme de l'Incarnation dans la théologie du père M.-D. Chenu ", Revue des Sciences philosophiques et théologiques, 69-3, p. 389-399.

Giroux Bernard, 2013, La Jeunesse étudiante chrétienne des origines aux années 1970, Paris, Éditions du Cerf.

Hervieu-Léger Danièle, 1993, La Religion pour mémoire, Paris, Éditions du Cerf.

Jossua Jean-Pierre, 1964, "Chrétien au monde, où en est la théologie de la "révision de vie" et de "l'événement" ?", Le Supplément de la vie spirituelle, 71, 1, p. 455-479.

Keck Thierry, 2013, "Maurice Montuclard: un intellectuel catholique? Contribution au débat sur la notion d'intellectuel catholique ", in A. Becker et al. (dir), Écrire l'histoire du christianisme contemporain, autour de l'œuvre d'Étienne Fouilloux, Paris, Karthala, p. 239-246.

L'Appel de la JOC, Paris, Librairie de la jeunesse ouvrière, s. d.

L'Action de l'aumônier, ce que les dirigeants jocistes attendent de leurs aumôniers, Paris, Librairie de la Jeunesse ouvrière, 1938.

Lebrun Jean, 1979, «L'héritage complexe de la JOC », Esprit, p. 67-77.

LEPRIEUR François, 1989, Quand Rome condamne: dominicains et prêtres ouvriers, Paris, Plon, Éditions du Cerf.

Manuel de la JOCF, 1929, Bruxelles, Secrétariat général de la JOC.

Muller Caroline, 2019, Au plus près des âmes et des corps. Une histoire intime des catholiques an XIX ${ }^{e}$ siècle, Paris, Presses universitaires de Frace.

Orieux Daniel, 1998, "Les Possibilités sociales de la foi: un parcours à travers le Dieu crucifié de Jürgen Moltmann et les débats de la Jeunesse Ouvrière Chrétienne dans les années 1975 ", mémoire de maîtrise de théologie, Université catholique de l'Ouest.

Pelletier Denis, 2002, La Crise catholique. Religion, société, politique en France (1965-1978), Paris, Payot.

Pennetier Claude, Pudal Bernard (dir.), 2002, Autobiographies, autocritiques, aveux dans le monde communiste, Paris, Belin.

Piette Albert, 1999, La Religion de près, Paris, Métailié. 
Pizivin Daniel, STRAsSer Robert (dir.), 2003, Croire, vivre, raconter: la révision de vie, une pratique à réinventer. Actes du colloque des 22-23 novembre 2002, Paris, Institut catholique de Paris.

Prudhomme Claude, 2012, «Les Jeunesses chrétiennes en crise », in D. Pelletier, J.-L. Schlegel (dir.), À la gauche du Christ, Paris, Éditions du Seuil, 2012, p. 323-340.

Raison du Cleuziou Yann, 2012, "Albert-Marie Besnard, un maître dépassé par ses disciples. Mystique de la génération et subversion des formes de l'autorité au couvent d'étude dominicain du Saulchoir (1964-1968) ", Genèses, 3, 88, p. 25-45.

Richou Françoise, 1997, La Jeunesse ouvrière chrétienne (JOC) : genèse d'une jeunesse militante, Paris, L'Harmattan.

Roche Bruno, Maincent Marie-Françoise, 2009, «Révision de vie ou relecture, pour faire quoi ? ", http://diaconat.catholique.fr/wp-content/uploads/sites/5/2014/12/ DA142.pdf [consulté le 23 avril 2019].

Routhier Gilles, 2012, “"Les signes des temps”. Fortune et infortune d'une expression du concile Vatican II ", Transversalités, 2, 118, p. 77-101.

SORET Jean-Hugues, 2007, Philosophies de l'Action catholique: Blondel-Maritain, Paris, Éditions du Cerf.

SORRel Christian (dir.), 2013, dossier «Les Matériaux Boulard, trente ans après. Des chiffres et des cartes, approches sérielles et spatiales en histoire religieuse ", Chrétiens et sociétés. Documents et mémoires, Lyon, LARHRA-RÉSÉA, 20.

Tranvouez Yvon, 2000, Catholiques et communistes. La crise du progressisme chrétien (1950-1955), Paris, Éditions du Cerf.

TuRINA Isacco, 2012, «Le Magistère post-conciliaire face au biopouvoir », in C. Béraud, F. Gugelot, I. Saint-Martin, Catholicisme en tensions, Paris, Éditions de l'EHESS, p. 191-202.

\section{La révision de vie. Une pratique religieuse méconnue au cœur du catholicisme français}

Cet article envisage la révision de vie comme une pratique importante $d u$ catholicisme français des années 1930 aux années 1980 pour la faire sortir de son relatif désintérêt dans la littérature scientifique actuelle. Si on peut percevoir la pratique comme étant l'un des ancêtres du "groupe de parole» contemporain, la révision de vie doit être également considérée dans sa dimension religieuse. Elle a pu répondre aux défis soulevés par le positionnement nouveau que souhaitaient avoir des militants, avec l'encouragement de l'institution, dans la société française d'après-guerre. Bien qu'elle n'ait pas eu les effets de reconquête religieuse initialement escomptés, qu'elle n'ait pas empêché la baisse de la pratique sacramentelle ni l'érosion des vocations religieuses et sacerdotales, la révision de vie a pu constituer un frein à la sécularisation en maintenant une présence religieuse dans des milieux réputés difficiles pastoralement. Peinant à s'adapter à la crise de la démocratie sociale et de la figure du militant qui y était associée, la pratique décline progressivement au cours des années 1970-1980. La révision de vie reflète donc un moment social et 
politique de la société française ainsi que la façon dont les groupes religieux, qui se réfèrent fortement à la tradition et à la continuité, sont en permanence acteurs d'innovations et témoins de mutations.

Mots-clés: révision de vie, Jeunesse ouvrière chrétienne, Jeunesse ouvrière chrétienne féminine, catholicisme, théologie du signe.

\section{The revision of life. An unrecognized religious practice at the heart of French Catholicism}

This article envisioned the revision of life as an important practice of French Catholicism from the 1930s to the 1980s to remove it from its relative lack of interest in the current scientific literature. If one can perceive the practice as being one of the ancestors of the contemporary "group of speech", the revision of life must also be considered in its religious dimension. It was able to respond to the challenges raised by the new positioning that militants wanted, with the encouragement of the institution, in post-war French society. Although it did not have the effects of religious reconquest initially expected, that it did not prevent the decline of the sacramental practice nor the erosion of the vocations religious and priestly, the revision of life could constitute a brake to secularization by maintaining a religious presence in environments deemed difficult pastorally. Painfully adapting to the crisis of social democracy and the figure of the activist who was associated with it, the practice gradually declined in the 1970s and 1980s. The revision of life thus reflects a social and political moment of French society as well as the way in which religious groups, which strongly refer to tradition and continuity, are constantly actors of innovations and witnesses of mutations.

Keywords: revision of life, Jeunesse ouvrière chrétienne, Jeunesse ouvrière chrétienne féminine, catholicism, theology of the sign.

\section{La revisión de vida. Una práctica religiosa desconocida en el corazón del catolicismo francés}

Este artículo considera la revisión de vida como una práctica importante del catolicismo francés desde la década de 1930 hasta la década de 1980 para eliminarla de su relativa falta de interés en la literatura cientifica actual. Si puede percibirse la práctica como uno de los ancestros del "grupo de apoyo" contemporáneo, la revisión de la vida también debe considerarse en su dimensión religiosa. Fue capaz de responder a los desafios planteados por el nuevo posicionamiento que los militantes querían, con el estímulo de la institución, en la sociedad francesa de posguerra. Aunque no tuvo los efectos de la reconquista religiosa que se esperaba inicialmente, que tampoco impidió el declive de la 
práctica sacramental ni la erosión de las vocaciones religiosas y sacerdotales, la revisión de la vida pudo constituir un freno a la secularización manteniendo una presencia religiosa en ambientes considerados pastoralmente difíciles. Adaptándose dolorosamente a la crisis de la socialdemocracia y de la figura del activista que se asociaba con ella, la práctica disminuyó gradualmente en los años 70 y 80. La revisión de vida refleja asi un momento social y político de la sociedad francesa, asi como la forma en que los grupos religiosos, que se refieren fuertemente a la tradición y a la continuidad, son constantemente actores de innovaciones y testigos de mutaciones.

Palabras clave: revisión de vida, Jeunesse ouvrière chrétienne, Jeunesse ouvrière chrétienne féminine, catolicismo, teología del signo. 